\title{
PROMOCIÓN INDUSTRIAL EN LA PROVINCIA DE JUJUY, ARGENTINA: ACTUANDO SOBRE EL DIAMANTE DE PORTER ${ }^{1}$
}

INDUSTRIAL PROMOTION IN THE PROVINCE OF JUJUY, ARGENTINA: ACTING OVER PORTER'S DIAMOND

JAIME ÍBER ALFARO ALIAGA VÍCTOR ADRIÁN LIPCHAK

UNIVERSIDAD NACIONAL DE JUJUY ARGENTINA. 


\title{
RESUMEN
}

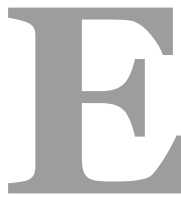

n este artículo se describe efectos de la Ley de Promoción Industrial vigente en la provincia de Jujuy, argumentando para verificar si las medidas promocionales existentes para el sector industrial, afectan de modo fundamentalmente diferente a los beneficiarios, de acuerdo a la naturaleza diferente de estos últimos, pudiendo por este motivo, favorecerse más a algunas situaciones de partida y modificaciones que a otras. También se desarrolla un análisis de la norma en su faceta de aplicación práctica, atendiendo a cuán esclarecedor es el Decreto Reglamentario, a la hora de hacer efectivas definiciones de la Ley. Los determinantes de la ventaja competitiva nacional, pueden devenir de ventajas competitivas creadas y no sólo heredadas. Así, la orientación de la inversión hacia combinaciones entre actividades, zonas geográficas y destinos específicos de inversión puede modificar el escenario competitivo. La norma se analiza en el sentido de apoyar su efectividad hacia esta meta.

Palabras clave: promoción industrial; clima de negocios; diamante de Porter.

\begin{abstract}
This article describes the effects of the current Industrial Promotion Act of the Province of Jujuy, in order to verify if this promotion measures affect in a fundamentally different way to beneficiaries, according to the differential nature of them, with the subsequent consequence of promoting some starting points and behaviors, over others. An analysis of the Act is also developed in its practical application, analyzing how illuminating is the Regulatory Decree, when definitions of the law go into the practical field. The determinants of national competitive advantage, can become from competitive advantages that are created and not just inherited. Thus, the orientation of investment to combinations of industries, geographical areas and specific investment destinations may change the competitive landscape. The effectiveness of this law toward this goal is analyzed in the article.
\end{abstract}

Keywords: industrial promotion; business climate; porter's Diamond. 


\section{INTRODUCCIÓN}

Es conocida la intención de los gobiernos a nivel nacional, provincial y municipal, de promover y dinamizar las actividades que mayor impacto tengan sobre el crecimiento y desarrollo, de la forma más eficiente posible.

En este sentido, como se plantea desde el Banco Interamericano de Desarrollo:

Numerosos estudios han demostrado que el sector privado constituye la fuerza impulsora del crecimiento económico, y es el más importante proveedor de actividad y oportunidades económicas para todos. En consecuencia, mejorar las condiciones para que el sector privado pueda realizar sus actividades resulta crucial para un crecimiento económico sostenido, es fundamental para los esfuerzos de reducción de la pobreza, y constituye un elemento esencial para el logro de los Objetivos de Desarrollo del Milenio.

Hay "consenso en que un clima de negocios favorable es aquél que proporciona a las empresas un sólido marco jurídico y normativo que promueva la competencia, fortalezca la gobernabilidad, permita superar las ineficiencias burocráticas y mejore el acceso a servicios financieros y de infraestructura. Un clima adecuado de negocios abarca desde la necesaria estabilidad en las políticas y en las reglas del juego que rigen la inversión, hasta la ausencia de barreras que obstaculizan el libre movimiento de capitales" (http://www.abc.com.py/, 2007).

Dentro de las múltiples acciones y sectores en los que las intenciones mencionadas en el primer párrafo, se convierten en hechos concretos (soportados por acciones, compromisos y presupuesto) las políticas de promoción industrial son un tipo de medida "tradicional", que en nuestro contexto, se han promovido con mayor intensidad y constancia que en otros países del continente, si analizamos el hecho desde su dimensión histórica.

Este tipo de incentivos, si se desarrollan dentro del contexto de políticas más amplias de mejoramiento clima de negocios, favorecen aspectos del llamado Diamante de Porter ya que, como sostiene Anzola (2013) “(Pueden) Servir como una rectificación de imperfecciones de mercado (...) especialmente en los mercados más pequeños distantes. (...). Pueden ser empleados para atraer fondos del exterior, para favorecer la transferencia de tecnología, o para influenciar decisiones de inversión hacia alguna industria o locación específica. Suelen incluir ventajas o reducciones impositivas, disponibilidad de infraestructura o capacidades de I+D. También pueden asociarse a concesiones de contratos gubernamentales y otras ventajas regulatorias"(p.23). De acuerdo a la UNCTAD (1996), "incluyen medidas específicas bien para incrementar la tasa de retorno de alguna inversión realizada, o para deducir o redistribuir sus costos o riesgos” (p.26).

La industria, presenta un alto atractivo como sector receptor de este tipo medidas, por su 
impacto no sólo directo -sobre la actividad promocionada- sino indirecto (sobre sus clientes, proveedores de insumos y servicios, etc.).

Es mayor su atractivo -además- por el modelo de "agregado de valor", ansiado por países tradicionalmente exportadores de materias primas como el nuestro, e importadores de bienes industrializados.

Las mencionadas acciones promocionales, en general, buscan un equilibrio al mejorar el clima de negocios, con normativas claras, accesibles y justas que identifiquen adecuadamente las necesidades de los potenciales inversores (sean proyectos nuevos o de ampliación), y cuidan no perjudicar de forma fundamental la dinámica competitiva, por ejemplo, hacia quienes ya se encuentran operando, y no acceden a estas medidas promocionales. Al mismo tiempo, tratan de "mostrar ventajas" relativas para que los inversores elijan una localización o emplazamiento en detrimento de otra.

La orientación de inversiones a combinaciones zona geográfica - actividades estratégicamente definidas, pueden reconvertir el panorama económico, razonando como Porter: hay ventajas que deben ser creadas, más allá de los recursos heredados. La formación de clusters industriales y de servicios a la industria, alrededor de alguna fuente natural o no de recursos, puede lograrse con un adecuado diseño y una eficiente implementación de leyes de promoción industrial, como la que analizamos.

Las investigaciones que se realizan sobre esta temática, pueden influenciar la toma de decisiones de los organismos ocupados de la implementación y de los legisladores que en sus mejores intenciones, pueden contar con información incompleta para decidir si una medida es o no adecuada, y sus efectos. Tal fue, la intención del proyecto de investigación en el que se originó este artículo.

El papel del gobierno a través de sus normativas de promoción industrial (que rebalancean las fuerzas e incentivos, buscando que las industrias se emplacen en ciertas localizaciones), incide en los factores asociados por Michael Porter a la ventaja competitiva, descritos en su modelo del "diamante". Explicaba el autor, respecto a uno de sus "Determinantes de la Ventaja Competitiva Nacional”:

"Es necesario que el sector privado desempeñe un papel en la creación de factores a fin de conseguir ventajas derivadas de los factores, en la mayoría de los sectores. Los factores avanzados y especializados son los más importantes para la ventaja competitiva, y nadie mejor que las empresas para saber cuáles son los más necesarios (...). Las inversiones públicas para la creación de factores normalmente se concentran en los factores más básicos y generalizados” (Porter, 1991, p. 122).

En la investigación que origina el presente artículo, desarrollamos la faz práctica (comprensión de posibles efectos de la aplicación de la Ley bajo estudio), y la faz teórica (relacio- 
nando el espíritu de la norma con ideas y corrientes de pensamiento económico). Parte de la comprensión de los posibles efectos prácticos, se describe en los apartados siguientes.

\section{OBJETIVO GENERAL}

Aportar elementos para evaluar los resultados de las medidas promocionales que favorecen a emprendimientos productivos nuevos y existentes, individuales y asociados, del sector industrial en la Provincia de Jujuy.

\section{OBJETIVOS ESPECÍFICOS}

3.1 Diagnosticar por medio de un modelo parametrizado y replicable, los efectos de las medidas promocionales sobre sectores de diferente naturaleza.

3.2 Evaluar el grado de utilidad (condicionado a la naturaleza del beneficia-rio) de las medidas de promoción industrial.

\section{IDENTIFICACIÓN DEL PROBLEMA}

Las normativas de promoción industrial vigentes en la provincia de Jujuy carecen de un sistema de análisis de efectos.

Particularmente, por ello, se desconoce cuál es el efecto diferenciado de estas medidas, dada la también diferente naturaleza de sus beneficiarios (sector, intensidad de capital/mano de obra, envergadura, uso de la capacidad instalada, distancia al centro de consumo, consumo de recursos, etc.).

\section{HIPÓTESIS}

Las medidas promocionales existentes para el sector industrial en la Provincia de Jujuy, afectan de modo fundamentalmente diferente a los beneficiarios, de acuerdo a la naturaleza de estos. 


\section{ANTECEDENTES}

El marco normativo es el que regula los derechos y obligaciones de, en este caso, los beneficiarios (empresas, inversores) de las normas de promoción industrial.

Las asimetrías regionales, originadas en múltiples causas (demográficas, geográficas, de infraestructura, etc.) hacen suponer que las medidas promocionales tomadas en pos de lograr el desarrollo de la actividad industrial, podrían ser "diferenciales", y "ajustadas a cada realidad regional".

Más aún, la distinta naturaleza de cada "sub - tipo" de actividad industrial, que difiere en intensidad de capital o mano de obra, en su tecnificación, momento dentro del ciclo de vida del producto, canales de comercialización, volúmenes de insumos, productos, o distancia a los centros de consumo, etc., podrían sugerir que medidas iguales, no tengan el mismo impacto sobre distintas regiones, y mucho menos lo tendrán sobre distintas actividades, o tamaño de empresa, por ejemplo.

Las características de las "Leyes de Promoción Industrial” (y su reglamentación) a lo largo de nuestro país, son bastante parecidas, a lo largo y ancho del país. Muchas similitudes, muchos textos idénticos.

Si bien esto puede entenderse parcialmente por la naturaleza "competitiva" de la norma (cada Provincia compite con otras por lograr la preferencia de radicación de las inversiones industriales, originando que se "empaten" algunos beneficios), también es dable creer que existen textos copiados - aunque este supuesto no es analizado en este artículo- sin un análisis profundo de los efectos que pueden ocasionar, una vez implementados.

Las políticas promocionales, requieren incluir un componente que las complemente: el análisis de sus efectos y la evaluación de resultados.

Por supuesto, quienes redactaron las normas mencionadas, asumen un buen resultado, y seguramente es esta la intención de cada letra plasmada en los proyectos de ley. Pero si quieren acercarse a un efecto atractivo para las inversiones (en este caso particular, orientadas a la industria), es necesario “(...) explicar por qué una (nación) brinda un entorno en el que las empresas mejoran e innovan y siguen haciéndolo más de prisa y con mejores orientaciones al compararlas con sus rivales (..)” (Porter, 1991, p. 109).

Es necesario, sin embargo, ver qué también estas normas han interpretado el contexto, la dinámica competitiva, las decisiones de inversión, la realidad, ya en territorio. Es necesario ver cómo se traducen tras su aplicación concreta, por ello elegimos armar modelos conceptuales que lleven hasta el límite lógico, la aplicación de la norma.

Tras el análisis de la letra de la norma, y el desarrollo de modelos conceptuales con los que se pueda verificar el grado de adaptación de las medidas promocionales, aceptación y factibilidad de su aplicación en distintos sectores, definiendo modelos de análisis aplicables y replicables, el presente artículo, desarrolla algunas de estas modelizaciones. 


\section{LA LEY 4392/88: DIFICULTADES PARA SU IMPLEMENTACIÓN}

El análisis hecho de la ley bajo estudio, ha tomado en cuenta la relación de los artículos de la misma con los de su Decreto Reglamentario (11298/89), y estudiado normas similares de otras provincias, o normas que actúan sobre otros aspectos (impositivo, por ejemplo), en el afán de encontrar cuán aplicable, comprensible y coherente es lo propuesto cono "beneficio promocional”, para los sujetos a los que quiere alcanzar.

\subsection{Aspectos Generales}

A lo largo de los diez capítulos de la ley y de los 56 anexos del Decreto Reglamentario (11298/89), es posible detectar inconsistencias, y definiciones confusas o ambiguas.

Se ha comparado conceptualmente estas normas con leyes y decretos de otras temáticas con el fin de compararlo con su forma de definir quién es el sujeto, la forma de determinar su pertenencia a una u otra clase, la ocasión, cuantía, forma de cálculo, criterio para excluir o exceptuar a alguien, etc.

Una de las más claras e inmediatas apreciaciones, es que estas normas, son casi idénticas en todo el país, hasta con similares errores de redacción: esto al menos hace pensar que se copia mucho, más que generar nuevas ideas. No fue nuestro objetivo analizar las posibles causas de esta práctica, pero conociéndola, se tiene una mejor perspectiva del ambiente en que se desarrolla la investigación.

\subsubsection{Sobre los beneficiarios}

El siguiente esquema, resume este aspecto de la ley, según los artículos específicos 5 y 7 .

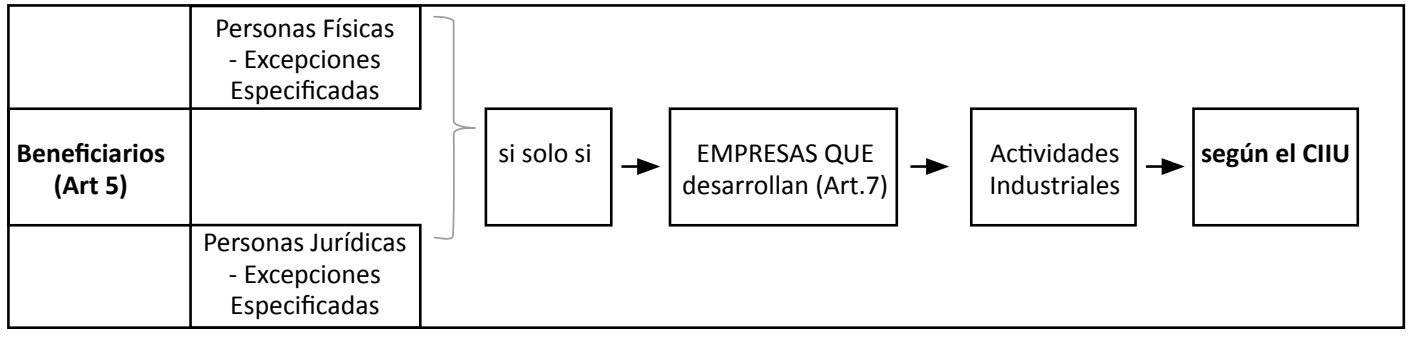

Fuente: Elaboración propia

Del artículo 5 se entiende: “TODAS las personas son beneficiarias potenciales”. Las únicas excepciones, son los expresamente impedidos por la norma. El Artículo 7 aclara la limitación: 
“ARTICULO 7: DE LA ACTIVIDAD INDUSTRIAL: Serán beneficiarios de esta ley, las empresas dedicadas a la actividad industrial que se instalen en el territorio de la Provincia. Se entenderá por actividad industrial aquella que logre la transformación física, química, o físico-química, en su forma o esencia, de materia prima o materiales, en nuevos productos a través de un proceso inducido mediante la aplicación de técnicas de producción uniforme, la utilización de maquinarias o equipos, la repetición de operaciones o procesos unitarios, llevada a cabo en un establecimiento industrial".

(Decreto Reglamentario) "De conformidad a la definición de actividad industrial establecida en el articulo 7 de la Ley, se considerara como tal aquellas actividades manufactureras que estén clasificadas y definidas en la CLASIFICACIÓN INTERNACIONAL UNIFORME DE TODAS LAS ACTIVIDADES ECONÓMICAS (C. I. I. U)”.

Sin embargo, analizando el contenido del resto de la norma, empezando por sus objetivos, es posible ver -en general- que las clasificaciones realizadas de beneficiarios y beneficios (ni sus intersecciones), no son mutuamente excluyentes ni colectivamente exhaustivas, ni dejan claro si desean serlo.

Como resultado, es bastante difícil determinar los criterios para listar a las potenciales beneficiarias de la ley, por ejemplo, no queda claro si el beneficiario de la medida promocional es:

* Una persona (física o jurídica)

* Un proyecto específico de inversión

* Un tipo de actividad puntual de una de estas personas

* La actividad con la que está registrada ante los entes recaudadores de tributos, o la que tiene por otras clasificaciones específicamente citadas en la norma.

De forma reiterada, se emplea la palabra "empresa", debiéndose recurrir a alguna jurisprudencia para entender el sentido dado a la misma, hecho que claramente afecta la transparencia y aplicabilidad de la norma, aumentando además, la discrecionalidad del órgano de aplicación, ya que "empresa" no es un tipo societario, ni un tipo de persona, por ejemplo.

\subsubsection{Ejemplos en los artículos, del uso de términos que dejan dudas sobre los beneficiarios:}

2 a) “(...) Promover el desarrollo de la actividad industrial (...)"

3 a) "Promover la instalación de industrias (...)"

3 b) "Propiciar el traslado de Fábricas"

3 c) “(...) desarrollo de actividades cooperativas (...)"

3 e) “(...) empresas de capital nacional (...)”

5 a) y b): Personas físicas, personas jurídicas

7 Empresas dedicadas a la actividad industrial 
Las combinaciones, los posibles solapamientos conceptuales, ayudan a multiplicar las opciones de interpretación. Por ejemplo: una sociedad (persona jurídica) con objeto social no industrial, inscrita ante organismos recaudadores con un código de actividad no industrial, dedicada al comercio, pero con un proyecto de inversión industrial (por ejemplo dedicarse a producir sus propios envases, actividad incluida en el CIIU) ¿es beneficiario? ¿Debería serlo? ¿Es posible impedir una presentación solicitando beneficios dela ley? ¿Cuál debería ser el peso relativo en las ventas, costos, uso de la capacidad, para que se considere industrial a todo el proyecto?

\subsection{De los requisitos para acceder a los beneficios}

También en la Ley y su Decreto, es posible encontrar inconsistencias o impericias técnicas en lo que se define como condiciones "operativas" para acceder a los beneficios promocionales.

Por ejemplo, la norma requiere que (Art 8 de la Ley, artículo 4 del decreto reglamentario) se realicen "ampliaciones de plantas industriales", que impliquen un $20 \%$ del valor de la inversión en activos fijos. Este porcentaje, calculado sobre el valor amortizado -o no- de tales activos tiene una sustancial diferencia, al poder pedirse "muy poco", si se toma el valor depreciado, o "demasiado", si se toma el valor de incorporación (esto abstrayéndonos de la posibilidad de períodos de inflación alta, o devaluaciones de la moneda).

Del mismo modo, ya desde la perspectiva operativa, el incremento medido en pesos, puede no tener nada que ver con la real incorporación de capacidad productiva: con un módulo adicional que complemente un recurso que opera como cuello de botella del sistema productivo de la empresa, quizá con sólo del $2 \%$ del valor de los activos fijos (que incluyen el terreno, instalaciones e inmueble, entre otros) es posible incrementar mucho la capacidad operativa.

Por motivos similares, tampoco es necesario pensar que sea imprescindible un $40 \%$ de incremento de capacidad teórica, si esta implica, para el inversor, una sobre estimación de la demanda potencial, dimensionando demás su planta, en aras de conseguir un beneficio fiscal parcial.

Las definiciones de la norma, que mezclan "actividad", "industria", "empresa", por ejemplo, pueden ser esquematizadas, de la siguiente manera (se incluyen definiciones de la ley, como "industria nueva" o "nueva empresa": 


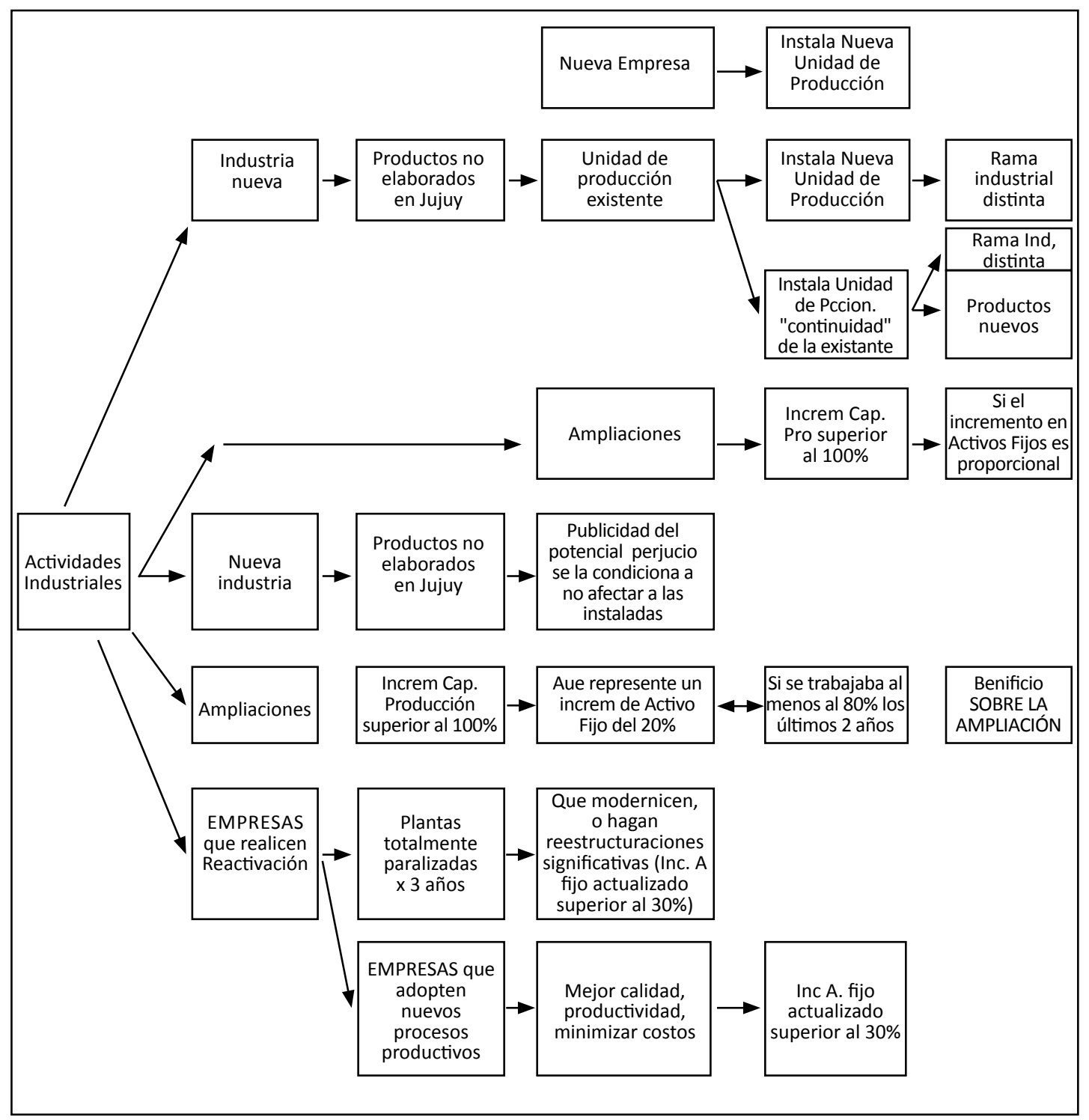

Fuente: Elaboración propia

\subsubsection{De los beneficios y su relación con los objetivos}

Por otro lado, en la citada ley se observa inconsistencia entre los objetivos incluidos en los primeros artículos (Art. 2; 3) y los beneficios realmente incorporados en el articulado de la Ley, o reglamentados en el decreto correspondiente. Se incluye en el siguiente esquema, resaltados, los objetivos sin correlato en beneficios: 


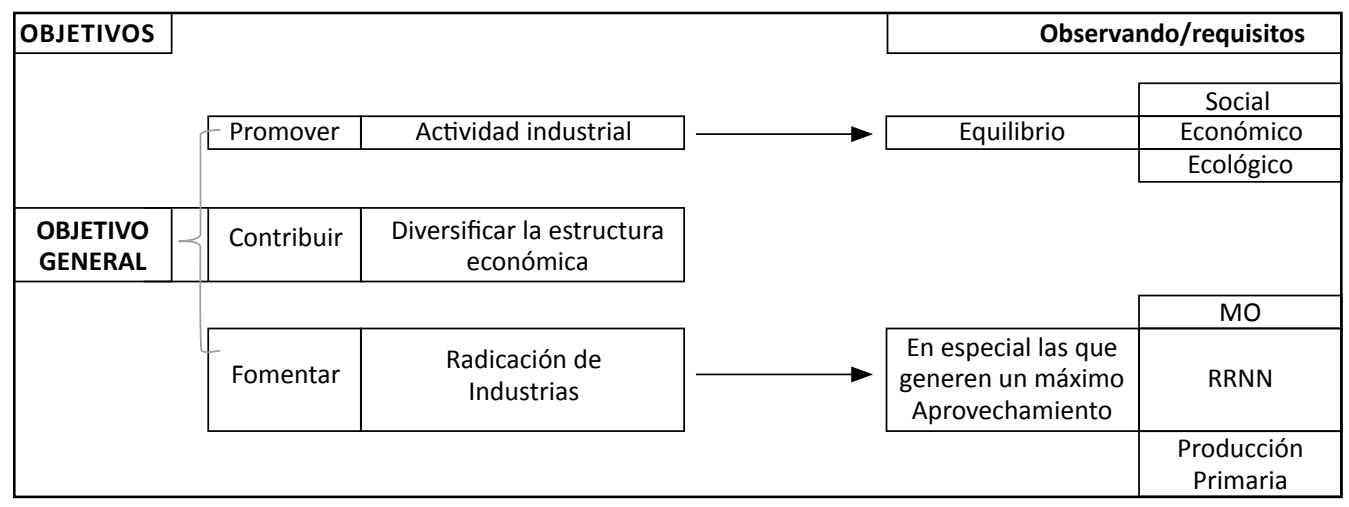

\begin{tabular}{|c|c|c|c|c|}
\hline & & $\begin{array}{c}\text { Instalación industrias } \\
\text { nuevas }\end{array}$ & & Alto desempleo \\
\hline & Promover & & Areas con & Bajo PBJ \\
\hline & & $\begin{array}{c}\text { Expansión industrias } \\
\text { existentes }\end{array}$ & & Alta migración \\
\hline OBJS. & & & Recursos & \\
\hline & Fomentar & Pleno empleo & Insumos & \\
\hline & & & MO Local & \\
\hline & Propender & Actividades Cooperativas & & \\
\hline
\end{tabular}

\begin{tabular}{|c|c|c|c|c|}
\hline & Propiciar & Traslado de fábricas & $\begin{array}{c}\text { a parques y zonas } \\
\text { industriales }\end{array}$ & \\
\hline & Reservar & $\begin{array}{l}\text { empresas de capital } \\
\text { nacional mayoritario }\end{array}$ & la prom ind & \\
\hline & Alentar & Desarrollado de la Pyme & & \\
\hline & Propender & $\begin{array}{l}\text { Participación de } \\
\text { empleadosen las Ut. }\end{array}$ & & \\
\hline & & & Cultural & \\
\hline \multirow[t]{6}{*}{\begin{tabular}{|c|} 
OBJS. \\
PARTICULARES \\
(CONT) \\
\end{tabular}} & Estimular & $\begin{array}{l}\text { Elevación de nivel } \\
\text { de personal }\end{array}$ & Técnico & \\
\hline & & & Sanitario & \\
\hline & Tender & $\begin{array}{c}\text { Consolidación de industrias } \\
\text { existantes }\end{array}$ & & $\begin{array}{l}\text { Sin dar poder } \\
\text { monopólico }\end{array}$ \\
\hline & Propender & $\begin{array}{l}\text { Incorporación de } \\
\text { tecnologías }\end{array}$ & & $\begin{array}{c}\text { Prioridad: origen } \\
\text { nacional }\end{array}$ \\
\hline & & & $\begin{array}{c}\text { Sustituyan } \\
\text { importaciones }\end{array}$ & \\
\hline & Incentivar & $\begin{array}{l}\text { Emprendimientos } \\
\text { Industriales }\end{array}$ & $\begin{array}{l}\text { Productos } \\
\text { exportables }\end{array}$ & \\
\hline
\end{tabular}

Fuente: Elaboración propia 


\subsubsection{De las actividades industriales incluidas}

Como parte del análisis, se ha estudiado los nomencladores / clasificadores que la ley y decreto mencionan, y se ha detectado que la apertura y codificación de la "Clasificación Industrial Internacional Uniforme de todas las Actividades Económicas” (CIIU)², documento de la División de Estadística (Departamento de Asuntos Económicos y Sociales - Naciones Unidas 2009) -tomada como parámetro de la Ley-, difiere de la empleada por normas nacionales: Clasificador Nacional de Actividades Económicas (CLANAE).

Esta diferencia complejiza la relación de los beneficios que la ley promueve, con las clasificaciones de otras normas, pongamos el ejemplo más crítico: Ley 25.924 de Promoción de Inversiones en Bienes de Capital y Obras de Infraestructura, y su decreto reglamentario (1152/2004). Esta norma, rige a nivel nacional, y emplea el CLANAE como nomenclador de actividades.

\subsection{Un resumen de los beneficios}

Ya mencionamos que parte de los objetivos no se correspondían con beneficios realmente incluidos en la norma, ahora enumeramos los artículos que otorgan beneficios similares, según cómo afectarían una decisión de inversión: los que reducen el monto de esta, los que reducen costos de funcionamiento (fijos o variables), las pre - financiaciones, etc.

A su vez, separamos los que son, en la práctica, aplicables (Reglamentados) y los que son sólo intenciones enunciadas:

\subsubsection{Beneficios reales:}

* Reducción en Costos Fijos : comodato en bienes del estado (10,b)

* Subsidio parcial a inversiones: Precio promocional de Bienes del Estado (10,b)

* Financiación Activos Fijos -Bienes Del Estado (10,b)

* Pre inversión: Asistencia y asesoramiento técnico (10,e)

* Reintegros (Reducción diferida de la Inversión): según el tipo de inversión y requisitos previos $(10, \mathrm{~h})$

* Administrativos: Apoyo oficial a gestiones de acogimiento a los beneficios previstos en la Ley Nacional $(10, \mathrm{~L})$

* Exenciones Impositivas ocasionales: Inscripción RPC, Imp. Sellos (11)

* Exenciones Impositivas periódicas: IIB, Impuesto inmobiliario (11)

* Reducción de tasas (11e)

${ }^{2}$ http://unstats.un.org/unsd/publication/seriesM/seriesm_4rev4s.pdf 


\section{7•3.2. Expresiones de deseo}

* Reducción de costos variables: Tarifa de energía eléctrica y otros prestados por la provincia $(10, a)$

* Prioridad en Licitaciones (10,c)

* Adjudicación de tierras fiscales (10, d)

* Construcción de vías de comunicación (10, e)

* Apoyo del Estado Provincial: agilización de los suministros, obtención de la protección arancelaria y fiscal, defensa a las contingencias del mercado externo, cualquier otra medida necesaria para mantener la planta industrial operando $(10, \mathrm{~g})$

* Procurar el apoyo oficial (10, i)

* Apoyo para la obtención de líneas de créditos de fomento $(10, \mathrm{j})$

* Toda colaboración, asesoramiento, gestión o intervención directa (10, k)

\section{EL ANÁLISIS DE LAS MEDIDAS PROMOCIONALES, DESDE SU EFECTO ECONÓMICO FINANCIERO}

$\mathrm{Al}$ estudiar la Ley, y tras identificar las diferentes empresas ${ }^{3}$ potenciales beneficiarias y caracterizar las que relevamos, desarrollamos varios modelos para el análisis de los efectos de la aplicación de las medidas de promoción, con la mayor simpleza conceptual posible, es decir, simplificando factores que pudieran resultar distorsivos / distractivos para el análisis.

En los ejemplos a continuación planteados podemos ver, en "empresas ficticias"-representadas por sus respectivos Estado de Situación Patrimonial y Estado de Resultados- cómo la medida promocional afecta de forma claramente diferente a distintos sujetos, de naturaleza y/o características diferentes.

De este modo, argumentamos para reforzar la línea de la Hipótesis de partida, que recordamos:

"Las medidas promocionales existentes para el sector industrial en la Provincia de Jujuy, afectan de modo fundamentalmente diferente a los beneficiarios, de acuerdo a la naturaleza de estos".

Incluimos fragmentos de Artículos de la Ley, y su Reglamentación respectiva, en cuanto a su interpretación y beneficio relacionado para cada caso planteado.

\footnotetext{
${ }^{3}$ Ya mencionamos la imprecisión de este término
} 


\subsection{Artículo 8 (Ley) actividades comprendidas:}

"Por la presente Ley se promueven las siguientes actividades: a) Industria de nuevos productos, no elaborados en la Provincia - industria nueva - que inicien sus actividades con posterioridad a la vigencia de la presente Ley";

ART. 4: (Decreto) "A los efectos del Inc. a) del artículo 8 de la Ley, se considera industrias de nuevos productos cuando:

(...)

Se instale una unidad de producción que posea continuidad física con otra existente de la misma empresa destinada a fabricar productos distintos de otra rama industrial y/o productos esencialmente diferentes. - Cuando se lleven a cabo ampliaciones que impliquen un incremento en la capacidad de producción superiores al $100 \%$, y como consecuencia de ello, un incremento proporcional en las inversiones activos fijos a realizar (...)”

ART.9 (Dto. Reglamentario): Exenciones impositivas

La exención de los impuestos previstos en el art. 11 de la Ley, podrá otorgarse teniendo en cuenta como valores máximos, las siguientes escalas de desgravación:

a) Para el caso de las actividades industriales comprendidas en los incisos a y b del art. $8^{\text {vo }}$. de la Ley:

\begin{tabular}{|c|c|}
\hline Años & Exención \\
\hline del $1^{\text {ro }}$ al $6^{\text {to }}$. & hasta un $100 \%$ \\
\hline el $7^{\text {mo }}$ & 85 \\
\hline el $8^{\text {vo }} 70$ & 70 \\
\hline el $9^{\text {no }} 55$ & 55 \\
\hline el $10^{\text {mo }} 40$ & 40 \\
\hline
\end{tabular}

Fuente: Ley 4392/88 (Jujuy)

\subsubsection{RELACIONANDO EL BENEFICIO CON LA CAPACIDAD INSTALADA Y LAS RESTRICCIONES DEL MERCADO}

Ejemplificando el caso, mostramos dos empresas de las que la primera (“A”) tiene una mayor cantidad de activos (duplica a la segunda - "B"-). En el modelo simplificado que se presenta, no existirán Bienes de Cambio (ni otros Activos Corrientes) y como Activo No Corriente, sólo existen Bienes de Uso.

Esta la situación en el momento T1 para ambas empresas ${ }^{4}$ :

\footnotetext{
${ }^{4}$ En el caso presentado, para ambas empresas (A y B) la financiación de la inversión, mantiene una proporción del 80\% para ambos casos, pero no se ha considerado para el análisis.
} 


\begin{tabular}{|c|c|c|}
\hline EMPRESA & A & DUPLICA ACTIVOS DE EMP. B \\
\hline
\end{tabular}

\begin{tabular}{|c|c|c|}
\hline T1 & & \\
\hline ACTIVO & & 100 \\
\hline ACTIVO CORRIENTE & & - \\
\hline Bienes de Cambio & - & \\
\hline ACTIVO NO CORRIENTE & & 100 \\
\hline BIENES DE USO & 100 & \\
\hline
\end{tabular}

\begin{tabular}{|c|c|c|}
\hline \multicolumn{2}{|c|}{ PASIVO } & $\mathbf{8 0}$ \\
\hline PASIVO CORRIENTE & - & - \\
\hline PASIVO NO CORRIENTES & & 80 \\
\hline & & \\
PROVEEDORES VS & 80 & \\
\hline \multicolumn{2}{|c|}{} & \\
\hline PATRIMONIO NETO & & $\mathbf{2 0}$ \\
\hline
\end{tabular}

EMPRESA B B $\quad 1$

$50 \%$ ACTIVOS DE A

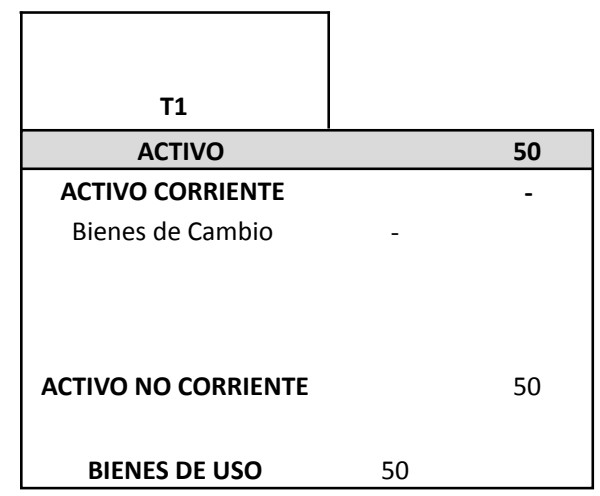

\begin{tabular}{|c|c|c|}
\hline \multicolumn{2}{|c|}{ PASIVO } & $\mathbf{4 0}$ \\
\hline PASIVO CORRIENTE & & - \\
\hline DS COMERCIALES & - & \\
\hline PASIVO NO CORRIENTES & & \\
\hline & & \\
PROVEEDORES VS & 40 & \\
\hline \multicolumn{2}{|c|}{} & \\
\hline PATRIMONIO NETO & & 10 \\
\hline
\end{tabular}

Fuente: Elaboración propia

Con el fin de argumentar el punto, para este y posteriores casos, los Activos Fijos se entenderán en un 100\% como bienes destinados a la producción. ${ }^{5}$

Este es el Estado de Resultados para las Empresa A y B, con restricciones de mercado (100 unidades) y un Costo de producción del 70\% de los ingresos por ventas. La alícuota de Impuesto a los Ingresos Brutos es de 2,5\%:

\footnotetext{
${ }^{5}$ Asumimos - también simplificando - una capacidad productiva que refleje una unidad de producción por unidad de tiempo, por cada peso de inversión en Activos Fijos.

La unidad de producción se produce de inmediato y se vende a \$1, también de inmediato si el mercado puede absorberlo, en efectivo. Las asunciones de liquidez inmediata, permiten acercar el Estadio de Resultados al análisis de Flujos de Fondos.
} 


\begin{tabular}{|lc|}
\hline \multicolumn{2}{|c|}{ A \$1 DE PRECIO DE VENTA LA UNIDAD, } \\
\hline \multicolumn{2}{|c|}{ EMPRESA } \\
\hline $\begin{array}{c}\text { CAP. PRODUCCIÓN } \\
\text { (FACTOR INV. EN } \\
\text { ACTIVOS FIJOS) }\end{array}$ \\
\hline CAPACIDAD DEL MERCADO & 1 \\
\hline ING X VENTAS & 100 \\
\hline COSTO DE PRODUCCIÓN & 100 \\
\hline UT NETA & 70 \\
\hline IIB (BENEFICIO) & $\mathbf{3 0}$ \\
\hline STOCK/ LEVERAGE OPERATIVO & $\mathbf{2 , 5 0}$ \\
\hline COSTOS DE INMOVILIZACIÓN & - \\
\hline FNF & - \\
\hline
\end{tabular}

Fuente: Elaboración propia

\begin{tabular}{|lc|}
\hline \multicolumn{1}{|c|}{ EMPRESA } & B \\
\hline $\begin{array}{c}\text { CAP. PRODUCCIÓN } \\
\text { (FACTOR INV. EN } \\
\text { ACTIVOS FIJOS) }\end{array}$ & \\
\hline CAP. DEL MERCADO & 1 \\
\hline ING X VENTAS & 100 \\
COSTO DE PRODUCCIÓN & 50 \\
\hline UT NETA & 35 \\
\hline IIB (BENEFICIO) & 15 \\
\hline STOCK/ LEVERAGE OPERATIVO & $\mathbf{1 , 2 5}$ \\
\hline COSTOS DE INMOVILIZACIÓN & -50 \\
\hline FNF & - \\
\hline
\end{tabular}

Fuente: Elaboración propia

Comparando ambos escenarios, la primera obvia diferencia que se observa es que la empresa con más activos (productivos), obtiene más ingresos y utilidad, aunque también está más expuesta a las limitaciones de mercado.

Como la empresa "B" tienen una capacidad inferior a la del mercado, tiene un efecto de "pérdida de oportunidad" en la magnitud de demanda de los productos, que no alcanza a cubrir (-50 unidades).

En la capacidad Inicial planteada, la primera firma ${ }^{6}$ obtendría de la Ley, el doble de Beneficio por la exención de Impuesto a los Ingresos Brutos por -también- obvias razones: produce $-\mathrm{y}$ vende- el doble.

Así, la empresa “A", estaría exenta de pago de\$2,50, mientras que la empresa B, sólo de $\$ 1,25$. Éste, el beneficio que obtendría por el régimen, montos no erogados de Impuesto.

\footnotetext{
${ }^{6}$ Firma, empresa, industria... seguimos en la redacción, con la ya mentada ambigüedad de la Ley.
} 
Ahora analizaremos cuál es el escenario, siguiendo los términos del artículo 8 de la Ley y 4 del Decreto Reglamentario, y evaluaremos las opciones que tendría un inversor, para elegir a cuál de las dos empresas le aporta capital.

La exigencia que representamos en el modelo respecto a la inversión fue incrementar en un 100\% la capacidad productiva (el mínimo exigido), para así obtener los 6 años de exención:

EMPRESA "A"

\begin{tabular}{|c|c|c|c|c|c|}
\hline \multicolumn{6}{|c|}{$\begin{array}{l}\text { ESTADO DE SITUACIÓN PATRIMONIAL EMPRESA "A", MOMENTO } 2 \\
\text { (POST INVERSIÓN) }\end{array}$} \\
\hline EMPRESA & A & \multicolumn{2}{|c|}{ MÁS ACTIVOS } & (momento 2) & \\
\hline T2 & $\begin{array}{c}\text { FACTOR } \\
\text { INCREMENTO A. } \\
\text { FIJO } \\
\end{array}$ & 2 & & & \\
\hline ACTIVO & & 200 & \multicolumn{2}{|l|}{ PASIVO } & 160 \\
\hline ACTIVO CORRIENTE & & - & \multicolumn{2}{|c|}{ PASIVO CORRIENTE } & - \\
\hline \multirow[t]{2}{*}{ BIENES DE CAMBIO } & - & & & - & \\
\hline & & & \multicolumn{2}{|c|}{ PASIVO NO CORRIENTE } & 160 \\
\hline ACTIVO NO CORRIENTE & & 200 & PROVEEDORES & 160 & \\
\hline BIENES DE USO & \multicolumn{2}{|l|}{200} & \multicolumn{2}{|c|}{ PATRIMONIO NETO } & 160 \\
\hline
\end{tabular}

\begin{tabular}{|c|c|c|c|}
\hline \multicolumn{4}{|c|}{$\begin{array}{l}\text { A } \$ 1 \text { DE PRECIO DE VENTA LA UNIDAD, } \\
\text { UNA UNIDAD PRODUCIDA POR CADA \$ DE Bs. DE USO }\end{array}$} \\
\hline EMPRESA & A & & \\
\hline CAP. PRODUCCIÓN & 1 & & \\
\hline CAP. MERCADO & 100 & & \\
\hline ING X VENTAS & 100 & \multirow{7}{*}{$\begin{array}{l}\text { A) DUPLICA INGRESOS Y MANTIENE } \\
\text { PROPORCIÓN EN COSTOS DE PRODUCCIÓN } \\
\text { B) MÁS EROGACIONES PORQUE APARECE } \\
\text { LA INMOVILIZACIÓN DE STOCK, HAY UNA } \\
\text { INVERSIÓN MAYOR } \\
\text { C) FNF AFECTADO POR EROGACIONES } \\
\text { EN INMOVILIZACIÓN, SUMADAS A } \\
\text { LOS COSTOS DE PRODUCCIÓN }\end{array}$} & \\
\hline COSTO DE PRODUCCIÓN & 70 & & \\
\hline UT NETA & 30 & & \\
\hline IIB (BENEFICIO) & 2,5 & & \\
\hline $\begin{array}{l}\text { STOCK/LEVERAGE } \\
\text { OBERATIVO }\end{array}$ & 100 & & $\begin{array}{c}\text { STOCK } \\
\text { INMOVILIZADO }\end{array}$ \\
\hline COSTO DE INMOVILIZACIÓN & 5,0 & & \\
\hline FNF & 25 & & \\
\hline
\end{tabular}

Fuente: Elaboración propia

Como se han duplicado las inversiones, todo el aporte en Activos Fijos (en nuestro ejemplo, reflejado en capacidad productiva) genera que el resultado se vea afectado negativamente por la existencia de restricciones de mercado: La empresa "A" empleando su capacidad pro- 
ductiva incremental (exigido por la norma para obtener el beneficio), tiene stock inmovilizado.

El stock que mencionamos tiene costos asociados económicos (por el costo de oportunidad, así como efectivamente erogados, como el costo de almacenamiento). En el caso, lo hemos ejemplificado como un $5 \%$ del valor del stock.

Analizando la empresa "B”, podemos ver esquemáticamente el resultado en el momento T2:

\begin{tabular}{|c|c|c|c|c|c|}
\hline \multicolumn{7}{|c|}{ ESTADO DE SITUACIÓN PATRIMONIAL EMPRESA "B", MOMENTO 2 } \\
(POST INVERSIÓN)
\end{tabular}

Fuente: Elaboración propia

\begin{tabular}{|l|r|}
\hline \multicolumn{2}{|c|}{ ESTADO DE RESULTADOS DE EMPRESA "B", MOMENTO 2} \\
(POST INVERSIÓN)
\end{tabular}

Fuente: Elaboración propia

La segunda empresa, con un inversión incremental de la mitad de la primera (en valores absolutos) obtiene un resultado, ventas y beneficio impositivo idénticos a la primera, porque si bien operan las restricciones del mercado, no le afectan, al estar su capacidad operativa -ya incrementada- por debajo o en el límite del mercado: No tiene stock inmovilizado, ni costo de 
inmovilización. Como resultado de esto, tiene menores erogaciones.

Un Flujo Neto de fondos mayor, ante inversiones iguales, daría un resultado mayor de VAN para la empresa "B”. Queda claro, además que en cualquier caso, ante una inversión mayor, se incrementará más la diferencia.

El beneficio así instrumentado, deviene en que la elección del inversor se orientaría a la firma que más lejos de su techo de mercado en un momento dado, y con una capacidad productiva dada.

Esto será cierto, siempre que el exceso de capacidad por sobre la demanda de mercado, sea usado en producción. Recordemos, que esta, es una condición requerida en el Decreto Reglamentario para obtener el beneficio.

\subsubsection{Relacionando los beneficios de la norma con la naturaleza de la inversión y la estructura productiva}

"ART. 11: En el caso de empresas existentes que realicen ampliaciones y/o reactivaciones los beneficios a otorgarse tendrán validez, por año calendario, a partir de la certificación por parte de la empresa de haber producido los volúmenes anuales superiores al cien por ciento (100\%) de la capacidad teórica existente anterior a la promoción acordada”.

A tal efecto las empresas promocionadas deberán presentar ante la Dirección Provincial de Industrias y Acción Cooperativa, al finalizar cada periodo anual de goce de beneficios, su declaración de Ingresos Brutos así como una declaración de los volúmenes producidos, y ventas anuales realizadas.

ARTICULO 8 (Ley) ACTIVIDADES COMPRENDIDAS: Por la presente Ley se promueven las siguientes actividades:

* (...) c) Ampliación de las plantas industriales ya existentes, cuando lo hagan como mínimo en un cuarenta por ciento (40\%) de capacidad de producción instalada y que comprenda a su vez el incremento de los activos fijos que posean en el momento de efectuar la solicitud correspondiente. Los beneficios a conceder lo serán en relación a la ampliación que se realice;

ART. 6: (Decreto Reglamentario) A los efectos del inciso c) del artículo 8 de la Ley, considerase ampliaciones de plantas industriales ya existentes cuando:

- Se incremente la capacidad de producción en un cuarenta por ciento (40\%) o más, siempre que se haya venido trabajando el ochenta por ciento (80\%) como mínimo de la capacidad teórica existente durante los dos ÚLTIMOS años, y que el aumento de la productividad sea el resultado de una inversión que incremente en por lo menos el veinte por ciento (20\%) las inversiones en activos fijos".

\subsubsection{Puntos de partida similares, diferencias post ampliación}

En este caso, nuevamente recurrimos a un modelo simplificado de empresas, que responden a la siguiente estructura patrimonial y de resultados: 
EMPRESA “A” (MANO DE OBRA INTENSIVA)

\begin{tabular}{|c|c|c|c|c|c|c|}
\hline \multicolumn{7}{|c|}{ ESTADO DE SITUACIÓN PATRIMONIAL T1 } \\
\hline \multirow[t]{7}{*}{ EMPRESA } & A & \multicolumn{5}{|c|}{ PREDOMINA MANO DE OBRA } \\
\hline & ACTIVO & & 3.000 & PASIVO & & 1.000 \\
\hline & ACTIVO CORRIENTE & & 2.000 & PASIVO CORRIENTE & & - \\
\hline & Bienes de Cambio & 2.000 & & & - & \\
\hline & & & & PASIVOS NO CORRIENTE & & 1.000 \\
\hline & ACTIVO NO CORRIENTE & & 1.000 & PROVEEDORES VS & 1.000 & \\
\hline & BIENES DE USO & 1.000 & & PATRIMONIO NETO & & 2.000 \\
\hline
\end{tabular}

Fuente: Elaboración propia

\begin{tabular}{|c|c|c|}
\hline \multicolumn{3}{|c|}{$\begin{array}{l}\text { ESTADO DE RESULTADOS } \\
\text { EMPRESA NO INTENSIVA T1 }\end{array}$} \\
\hline \multicolumn{3}{|c|}{$\begin{array}{l}\text { A \$1 DE PRECIO DE VENTA LA UNIDAD, } \\
\text { SE ASUMEN GIF EFECTIVAMENTE EROGADOS } \\
\text { (NO AMORTIZACIONES, POR EJEMPLO) }\end{array}$} \\
\hline ING X VENTAS & & 100 \\
\hline COSTO DE PRODUCCIÓN & & 80 \\
\hline M. OBRA & 20 & \\
\hline M. PRIMA & 40 & \\
\hline GIF & 20 & \\
\hline UT NETA & & 20 \\
\hline IIB (BENEFICIO) & & 2,5 \\
\hline UTILIDAD/VENTAS & & 0,20 \\
\hline
\end{tabular}

Fuente: Elaboración propia

\section{EMPRESA “B” (CAPITAL INTENSIVA)}

\begin{tabular}{|c|c|c|c|c|c|}
\hline \multicolumn{6}{|c|}{ ESTADO DE SITUACIÓN PATRIMONIAL T1 } \\
\hline EMPRESA & B & \multicolumn{4}{|c|}{$\begin{array}{l}\text { PREDOMINA MÁQUINAS } \\
\text { (CAPITAL INTENSIVA) }\end{array}$} \\
\hline ACTIVO & & 3.000 & PASIVO & & 1.000 \\
\hline ACTIVO CORRIENTE & & 2.000 & PASIVO CORRIENTE & & - \\
\hline \multirow[t]{2}{*}{ Bienes de Cambio } & 2.000 & & & - & \\
\hline & & & PASIVOS NO CORRIENTE & & 1.000 \\
\hline ACTIVO NO CORRIENTE & & 1.000 & PROVEEDORES VS & 1.000 & \\
\hline BIENES DE USO & 1.000 & & PATRIMONIO NETO & & 2.000 \\
\hline
\end{tabular}

Fuente: Elaboración propia 


\begin{tabular}{|c|c|c|}
\hline \multicolumn{3}{|c|}{$\begin{array}{l}\text { ESTADO DE RESULTADOS } \\
\text { EMPRESA CAPITAL INTENSIVA }\end{array}$} \\
\hline \multicolumn{3}{|c|}{$\begin{array}{l}\text { A } \$ 1 \text { DE PRECIO DE VENTA LA UNIDAD, } \\
\text { SE ASUMEN GIF EFECTIVAMENTE EROGADOS } \\
\text { (NO AMORTIZACIONES, POR EJEMPLO) }\end{array}$} \\
\hline \multicolumn{2}{|l|}{ ING X VENTAS } & 100 \\
\hline \multicolumn{2}{|c|}{ COSTO DE PRODUCCIÓN } & 80 \\
\hline M. OBRA & 20 & \\
\hline M. PRIMA & 40 & \\
\hline GIF & 20 & \\
\hline \multicolumn{2}{|l|}{ UT NETA } & 20 \\
\hline IIB (BENEFICIO) & & 2,5 \\
\hline
\end{tabular}

Fuente: Elaboración propia

En este caso, hemos elegido patrimonios idénticos al momento T1. La diferencia operativa se puede observar en los costos de producción, una vez producido el incremento en las inversiones, de acuerdo a lo exigido por la norma (20\% de los activos productivos):

\section{EMPRESA “A” (MANO DE OBRA INTENSIVA)}

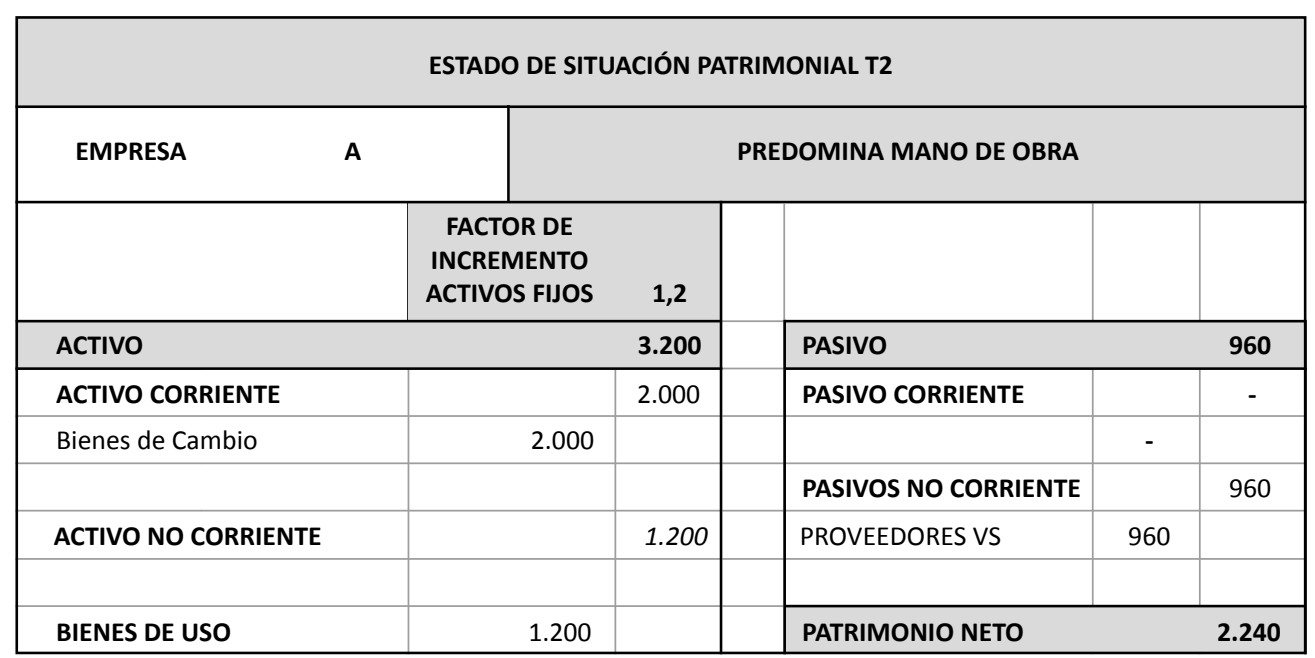

Fuente: Elaboración propia 


\begin{tabular}{|l|c|c|c|}
\hline \multicolumn{3}{|c|}{$\begin{array}{c}\text { ESTADO DE RESULTADOS } \\
\text { EMPRESA NO INTENSIVA T2 }\end{array}$} \\
\hline & $\begin{array}{c}\text { FACTOR DE } \\
\text { INCREMENTO } \\
\text { ACTIVOS FIJOS }\end{array}$ & $\mathbf{2}$ & $\begin{array}{c}\text { FACTOR DE } \\
\text { INCREMENTO POR SUB } \\
\text { RUBRO DEL COSTO }\end{array}$ \\
\hline ING X VENTAS & $\mathbf{2 0 0}$ & \\
\hline COSTO DE PRODUCCIÓN & 140 & \\
\hline M. OBRA & 40 & & 2 \\
\hline M. PRIMA & 80 & & 2 \\
\hline GIF & 20 & & 1 \\
\hline UT NETA & & $\mathbf{6 0}$ & \\
\hline IIB (BENEFICIO) & & $\mathbf{5 , 0}$ & \\
\hline UTILIDAD/VENTAS & $\mathbf{0 , 3 0}$ & \\
\hline
\end{tabular}

Fuente: Elaboración propia

La respuesta a cuál empresa tendrá un mayor Flujo Neto de Fondos, está definida por la preponderancia del capital sobre la mano de obra en la composición de los costos (Pudiendo ser por la naturaleza distinta de la actividad de las empresas "A" y "B", o ante similares actividades, simplemente distinta tecnología empleada para producir).

La empresa más tecnificada tiene puede incrementar su producción incurriendo en costos variables por mano de obra ${ }^{7}$ menores a los de su par orientada a la mano de obra. Como mostramos con el ejemplo, la primera obtendrá un mayor Flujo Neto de Fondos.

Nuevamente, vemos que la norma favorecerá más a un tipo de empresa que a otras: la empresa que más lejos se halle del límite de su leverage operativo, es la más beneficiada. Esto es, en empresas con diferente estructura productiva (y de costos) debido a que la inversión incremental exigida en activos fijos productivos, combinada con la condición de que esta inversión se encuentre efectivamente produciendo, hacen que -con un mismo nivel de ingresos por ventas-, la empresa más tecnificada incurra en menores costos variables (es decir, erogaciones efectivas: un mayor Flujo Neto de Fondos).

\subsubsection{Puntos de partida diferente, diferencia en la ampliación}

Ajustando el ejemplo anterior, podemos pensar que la empresa más tecnificada, tendría desde el inicio, un nivel de inversión mayor respecto a la menos tecnificada, pudiendo este efecto compensar los Flujos de Fondos Futuros, que como mostramos en el apartado anterior, son mayores cuanto más lejos se halle la empresa de su límite de capacidad.

\footnotetext{
${ }_{7}$ Para ambos casos, asumimos que el costo de materia prima incremental y gastos indirectos de fabricación, es idéntico, efectivamente erogado, y que crece en proporción al crecimiento en la producción.
} 
Planteamos entonces el siguiente escenario, idéntico para la empresa Mano de Obra Intensiva del punto anterior (en ambos mostramos además las relaciones de costos de producción dado su respectiva capacidad operativa):

\section{EMPRESA “A” (MANO DE OBRA INTENSIVA)}

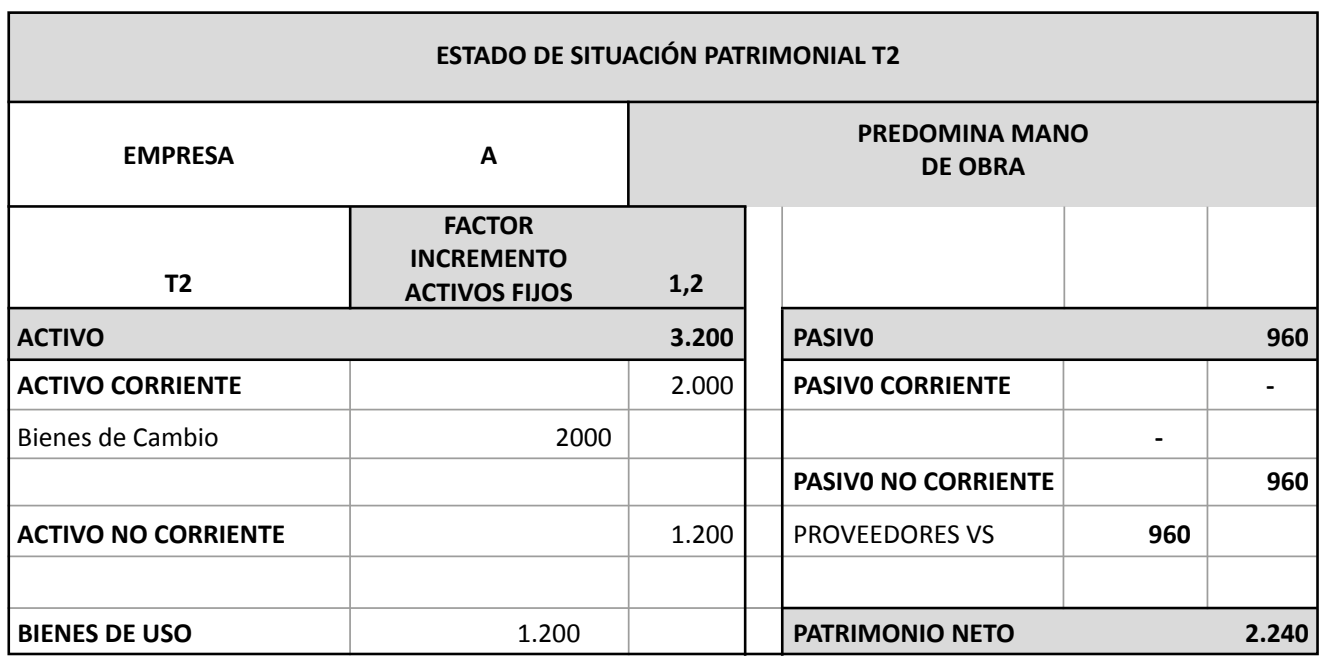

Fuente: Elaboración propia

\begin{tabular}{|l|c|c|c|}
\hline \multicolumn{3}{|c|}{$\begin{array}{c}\text { ESTADO DE RESULTADOS } \\
\text { EMPRESA NO INTENSIVA T2 }\end{array}$} \\
\hline & $\begin{array}{c}\text { FACTOR } \\
\text { INCREMENTO } \\
\text { PRODUCCIÓN }\end{array}$ & 2 & $\begin{array}{c}\text { FACTOR DE } \\
\text { INCREMENTO POR SUB } \\
\text { RUBRO DEL COSTO }\end{array}$ \\
\hline ING X VENTAS & $\mathbf{2 0 0}$ & \\
\hline COSTO DE PRODUCCIÓN & 140 & 2 \\
\hline M. OBRA & 40 & & 2 \\
\hline M. PRIMA & 80 & 1 \\
\hline GIF & 20 & & \\
\hline UT NETA & & 60 & \\
\hline IIB (BENEFICIO) & & $\mathbf{0 , 3 0}$ & \\
\hline UTILIDAD/VENTAS & & \\
\hline
\end{tabular}

Fuente: Elaboración propia 
EMPRESA "B" (CAPITAL INTENSIVA - MAYORES ACTIVOS)

\begin{tabular}{|c|c|c|c|c|c|c|}
\hline \multicolumn{7}{|c|}{ ESTADO DE SITUACIÓN PATRIMONIAL T1 } \\
\hline \multirow[t]{7}{*}{ EMPRESA } & B & \multicolumn{5}{|c|}{$\begin{array}{c}\text { PREDOMINAN MÁQUINAS } \\
\text { (CAPITAL INTENSIVA - MAYORES ACTIVOS) }\end{array}$} \\
\hline & \multicolumn{2}{|l|}{ ACTIVO } & 7.000 & \multicolumn{2}{|l|}{ PASIVO } & 1.000 \\
\hline & ACTIVO CORRIENTE & & 2.000 & PASIVO CORRIENTE & & - \\
\hline & Bienes de Cambio & 2000 & & & - & \\
\hline & & & & PASIVO NO CORRIENTE & & 1.000 \\
\hline & ACTIVO NO CORRIENTE & & 5.000 & PROVEEDORES VS & 1.000 & \\
\hline & BIENES DE USO & 5.000 & & PATRIMONIO NETO & & 6.000 \\
\hline
\end{tabular}

Fuente: Elaboración propia

\begin{tabular}{|c|c|c|}
\hline \multicolumn{3}{|c|}{$\begin{array}{l}\text { ESTADO DE RESULTADOS } \\
\text { EMPRESA CAPITAL INTENSIVA T1 }\end{array}$} \\
\hline \multicolumn{3}{|c|}{$\begin{array}{l}\text { A \$1 DE PRECIO DE VENTA LA UNIDAD, } \\
\text { SE ASUMEN GIF EFECTIVAMENTE EROGADOS } \\
\text { (NO AMORTIZACIONES, POR EJEMPLO) }\end{array}$} \\
\hline \multicolumn{2}{|l|}{ ING $X$ VENTAS } & 500 \\
\hline \multicolumn{2}{|c|}{ COSTO DE PRODUCCIÓN } & 320 \\
\hline M. OBRA & 20 & \\
\hline M. PRIMA & 200 & \\
\hline GIF & 100 & \\
\hline \multicolumn{2}{|l|}{ UT NETA } & 180 \\
\hline IIB (BENEFICIO) & & 12,5 \\
\hline UTILIDAD/VENTAS & & 0,36 \\
\hline
\end{tabular}

Fuente: Elaboración propia

Como puede verse, es razonable tener una producción mayor dada una mayor inversión en activos productivos. En ambos ejemplos mantuvimos una relación del 10\% de productos elaborados y vendidos por cada peso de Activos Fijos Disponibles.

En la empresa de mayor tecnificación, la Utilidad es mayor (en términos absolutos y relativos), al tener un costo de mano de obra menor.

$\mathrm{Si}$ a este escenario se le aplica el requerimiento normativo del incremento en activos productivos de un $20 \%$, tenemos el siguiente resultado: 
EMPRESA “B” (CAPITAL INTENSIVA) - AMPLIANDO LA INVERSIÓN

\begin{tabular}{|c|c|c|c|c|c|}
\hline \multicolumn{6}{|c|}{$\begin{array}{l}\text { ESTADO DE SITUACIÓN PATRIMONIAL T2 PREDOMINAN LAS MÁQUINAS } \\
\text { (CAPITAL INTENSIVA - MAYORES ACTIVOS) }\end{array}$} \\
\hline & $\begin{array}{l}\text { FACTOR DE INCREMENTO } \\
\text { ACTIVOS FIJOS }\end{array}$ & 1,2 & & & \\
\hline ACTIVO & & 8.000 & PASIVO & & 4.800 \\
\hline ACTIVO CORRIENTE & & 2.000 & PASIVO CORRIENTE & & - \\
\hline \multirow[t]{2}{*}{ Bienes de Cambio } & 2000 & & & - & \\
\hline & & & PASIVO NO CORRIENTE & & 4.800 \\
\hline ACTIVO NO CORRIENTE & & 6.000 & PROVEEDORES VS & 4.800 & \\
\hline BIENES DE USO & 6.000 & & PATRIMONIO NETO & & 3.200 \\
\hline
\end{tabular}

Fuente: Elaboración propia

\begin{tabular}{|c|c|c|c|}
\hline \multicolumn{3}{|c|}{$\begin{array}{l}\text { ESTADO DE RESULTADOS } \\
\text { EMPRESA NO INTENSIVA T2 }\end{array}$} & \multirow[b]{2}{*}{$\begin{array}{c}\text { FACTOR DE } \\
\text { INCREMENTO POR SUB } \\
\text { RUBRO DEL COSTO }\end{array}$} \\
\hline & $\begin{array}{l}\text { FACTOR DE } \\
\text { INCREMENTO } \\
\text { PRODUCCIÓN }\end{array}$ & 2 & \\
\hline \multicolumn{2}{|l|}{ ING $X$ VENTAS } & 1.000 & \\
\hline \multicolumn{2}{|c|}{ COSTO DE PRODUCCIÓN } & 620 & \\
\hline M. OBRA & 20 & & 1 \\
\hline M. PRIMA & 400 & & 2 \\
\hline GIF & 200 & & 2 \\
\hline \multicolumn{2}{|l|}{ UT NETA } & 380 & \\
\hline IIB (BENEFICIO) & & 25,0 & \\
\hline \multicolumn{2}{|c|}{ UTILIDAD/VENTAS } & 0,38 & \\
\hline
\end{tabular}

Fuente: Elaboración propia

Como puede observarse, el incremento -en tanto la empresa tecnificada se halle dentro de sus límites de leverage operativo- reportará una utilidad mayor -obviamente en términos absolutos- pero también en términos relativos.

Los incrementos del 20\% en la inversión, para cada caso, significaron:

* Empresa A: \$200

* Empresa B: $\$ 1.000$

* Inversión Diferencial de B sobre A: $\$ 800$ 
Tras ver este escenario, nos preguntamos si las cifras arbitrariamente elegidas, tienen un punto o nivel de inversión en el que se puedan considerar efectos diferentes, ya que los Flujos Netos de Fondos, serán mayores en el segundo caso (empresa tecnificada), pero la inversión requerida es mayor.

El equilibrio (punto de indiferencia entre los dos tipos de empresa) se da cuando la inversión diferencial, se iguala al VAN Diferencial de los Flujos netos de Fondos de ambas opciones.

Esto quiere decir que calculando la Inversión en exceso que la empresa más tecnificada requiera por sobre la mano de obra intensiva, tengo el valor que la diferencia entre los Flujos Netos de la opción tecnificada y la de mano de obra intensiva deben alcanzar, para hacer que la más tecnificada sea más conveniente.

En todas las demás combinaciones, la medida beneficia más a la empresa con mayor leverage operativo, por la naturaleza de su inversión, orientada a la tecnología más que a la mano de obra como factor productivo predominante.

Lo que se demuestra en este análisis, es que la norma no resulta indiferente a la condición de mano de obra intensiva o capital intensiva, y no es indiferente al apalancamiento operativo disponible, excepto en un único punto de indiferencia. Ergo, la norma tiene esencialmente, beneficios diferentes para empresas de distinta naturaleza.

\section{CONCLUSIONES}

Elegimos como objeto de estudio a un instrumento por medio del cual se promueve el desarrollo industrial, como medio para -en algún sentido cuali o cuantitativo- mejorar la realidad provincial, afectando algunos de los componentes del Diamante de Porter.

Si las acciones de política económica, como esta norma, pretenden movilizar fuerzas del mercado -las locacionales, por ejemplo- para redireccionar cierto tipo de inversiones que "naturalmente" podrían elegir otros emplazamientos, hallamos importante reconocer tanto los vacíos conceptuales de los que adolece aquélla, como también las falencias prácticas que presenta, implicando en ocasiones, que buenas intenciones queden sin reglamentar, o peor aún, que al reglamentar mal conceptos que serían de gran utilidad, los errores la hagan devenir en ineficiente.

En ese marco - como describimos en los primeros apartados- realizamos un análisis de la Ley y su Decreto Reglamentario encontrando serias falencias en las definiciones que hacen a la esencia misma de la norma, y que la alejan de su mejor efecto, por la confusión que generan. También pudimos evidenciar vacíos reglamentarios que impiden la operativización de beneficios que pudieron ser de interés para el sector industrial. Hallamos también impericias técnicas en las definiciones, desnudando posibles efectos negativos en las explotaciones, en el caso de realizar el tipo de inversiones que la norma pide para otorgar los beneficios. 
Al plantearnos el proyecto de investigación que origina el presente artículo, entendíamos que las normativas de promoción industrial vigentes en la Provincia de Jujuy serían más eficientes y efectivas, de contar un sistema de análisis de efectos en territorio. En la segunda parte del trabajo, nos abocamos a este análisis.

De los efectos posibles nuestra preocupación se enfocó particularmente en que se desconocía cuál era el efecto de las medidas promocionales, dada la diferente naturaleza de sus beneficiarios (sector industrial, intensidad de capital/mano de obra, envergadura, uso de la capacidad instalada, distancia al centro de consumo, consumo de recursos, etc.), por ello nuestro punto de partida, fue la hipótesis de que las medidas promocionales existentes para el sector industrial en la Provincia de Jujuy, afectan de modo fundamentalmente diferente a los beneficiarios, de acuerdo a la naturaleza diferente de estos últimos.

Como en el apartado final mostramos, esta hipótesis es verdadera, ya que desde puntos de partida similares (representados por estructuras patrimoniales y de resultados), los efectos pueden diferir mucho al producirse alguna de las condiciones que la ley exige (por ejemplo, un cierto nivel de inversiones). Del mismo modo, situaciones de partida diferentes, condicionaban la magnitud de los beneficios que pudieran obtenerse, no tanto por lo que la ley prometía (exenciones de impuestos esencialmente), sino por el efecto en los resultados de la propia actividad que se desea promocionar.

Aspectos no evaluados en el presente trabajo (como restricciones de acceso a factores, mercados, o el costo de la deuda) combinados con las múltiples opciones y condiciones propuestas por la norma, generan muchas posibles combinaciones de escenarios de partida diferentes, en las que una medida estándar puede afectar a empresas de diferente naturaleza y características.

\section{REFERENCIAS BIBLIOGRÁFICAS}

Anzola, M. (2013). Framework For Private Sector Development In Suriname Development Of An Action Plan For Business Climate Reform Priorities.

Clima de negocios. (07 de Junio de 2007). Obtenido de: www.abc.com.py/edicion-impresa/ opinion/clima-de-negocios-986765.html

Cámara de diputados de la Provincia de Jujuy. (1989). Ley 4392/88 de Promoción Industrial.

Poder Ejecutivo de la Provincia de Jujuy. (1989). Decreto 11298 Reglamentario de la Ley 4392 de Promocion Industrial. 
Porter, M. (1991). La Ventaja Competitiva de las Naciones. Buenos Aires: Vergara.

UNCTAD. (2015, Julio 25). Incentives and Foreign Direct Investment" Division of Transnational Corporations and Investment. Retrieved from http://idbdocs.iadb.org/wsdocs/ getDocument.aspx?DOCNUM=38571795

\section{CURRICULUM VITAE}

\section{Jaime Íber Alfaro Aliaga}

Doctorando en Ciencias Sociales, Maestrando en Teoría y Metodología de las Ciencias Sociales, Universidad Nacional de Jujuy (UNJu). Master Ejecutivo en Dirección de Empresas Fundación Del Tucumán - Pontificia Universidad Católica de Valparaíso. Licenciado en Administración de Empresas (UNJu), Actualmente Director del Instituto de Investigaciones de la Facultad de Ciencias Económicas de la UNJu (FCE, UNJu). Profesor Adjunto de Investigación de Mercados, Quinto año de la Licenciatura en Administración de la FCE - UNJu. Miembro del Directorio del Consejo de la Microempresa de la Provincia de Jujuy (Argentina). Socio de Lúmino Consultores de Negocios. Fue miembro del Equipo Técnico de la Dirección Provincial de Desarrollo Industrial y Comercial de la Provincia de Jujuy.

jaimeiber@hotmail.com

\section{Víctor Adrián Licphak}

Maestrando en Teoría y Metodología de las Ciencias Sociales, Universidad Nacional de Jujuy (UNJu). Master Ejecutivo en Dirección de Empresas Fundación Del Tucumán - Pontificia Universidad Católica de Valparaíso. Ingeniero Agrónomo, Universidad Nacional de Tucumán (UNT). Fue Director de la Dirección Provincial de Desarrollo Industrial y Comercial de la Provincia de Jujuy, Director del Instituto de Calidad de la Provincia de Jujuy, en representación del Ministerio de Producción y Medio Ambiente, Miembro del equipo técnico de Equipo Técnico de la Fundación Jujeña para el Desarrollo Sustentable adlipchak@gmail.com 